stellten geringen Schichtenmächtigkeit der baumlosen Tundrenzeit zu urteilen, offenbar nicht über das Gotiglazial zurückreichen; bis dahin kann die pollenanalytisch erfaßbare Sedimentation nur durch Erdfließen verhindert worden sein. $\mathrm{Da}$ die Klimadepression zur Zeit des mittelschwedischen Eisrandgürtels (also in der jüngeren Dryas-Zeit) bis in die Alpen hinein in den Schichtenfolgen und Pollendiagrammen so deutlich erkennbar ist, müßte der Langeland-Vorstoß (= Bildung der gotiglazialen Anfangsmoräne) stratigraphisch und pollenanalytisch erst recht deutlich nachweisbar sein (was aber nicht der Fall ist), wenn in Deutschland die Pollendiagramme über das Gotglazial hinaus zurückreichen würden.

Das Verdienst, die Möglichkeit entdeckt und bewiesen zu haben, mit Hilfe vulkanischer Schichten exakte geologische Datierungen auszuführen, gebührt V. AUER (vergl. seine zusammenfassende Arbeit von 1948).

$\mathrm{Schriftenverzeichnis}$

AHRENS, W. und STEINBERG, K. (1943): Jungdiluvialer Tuff im Eichsfeld. Ber. Reichsamt f. Bodenforschung Jahrg. 1943.

ANDREE, J. (1939) Der eiszeitliche Mensch in Deutschland. - Stuttgart.

AUER, V. (1948) Las capas volcánicas como nuevo método de cronologia postglazial en Fuegopatagonia.- Gaea (Buenos Aires) 8, S. 311-336.

BANDI, H.-G. (1947) Die Schweiz in der Rentierzeit. Frauenfeld.

BEHLEN, H. (1905) Das Alter und die Lagerung des Westerwälder Bimssandes. Jahrb. d. Nassauischen Vereins f. Naturk. 58, S. 44-51.

FIRBAS, F. (1935) Die Vegetationsentwicklung des mitteleuropäischen Spätglazials. Bibliotheca Botanica H. 112.

GÜNTHER, A. (1924) Vulkantätigkeit und Eiszeit im östlichen Eifelvorland zwischen Mosel und Vinxtbach. - „Die Eiszeit“ 1. S. 46-59.

HECK, H. (1925) Das rheinische Paläolithikum. - „Die Eiszeit“ 2, S. 96-111.

KRÅUSEL, R. und WEYLAND, H. (1942) Tertiäre und quartäre Pflanzenreste aus den vulkanischen Tuffen der Eifel. - Abh. senckenberg. naturf. Ges. Nr. 463, S. $1-62$.

RUST, A. (1943) Die alt- und mittelsteinzeitlichen Funde von Stellmoor. - Neumünster. - (1948) Jungpaläolithische Wohnanlagen bei Hamburg. — „Hammaburg" 1 , S. 33-38.

SCHAAFFHAUSEN, H. (1888) Die vorgeschichtliche Ansiedlung in Andernach. Jahrbücher d. Ver. v. Altertumsfreunden im Rheinlande 86, S. 1-41, Bonn.

SCHWABEDISSEN, H. (1944) Die mittlere Steinzeit im westlichen Norddeutschland. Neumünster. - (1949) Hamburg-Rissen, ein wichtiger Fundplatz der frühen Menschheitsgeschichte. - „Hammaburg“ 2, S. 81-90.

WOLDSTEDT, P. (1929) Das Eiszeitalter. - Stuttgart. - (1935) Die Beziehungen zwischen den nordischen Vereisungen und den paläolithischen Stationen von Nord- und Mitteldeutschland. - „Mannus“ 27, S. 275-287.

\title{
Australopithecus oder Plesianthropus oder Paranthropus?
}

\section{Von Hans Weinert, Kiel}

Als 1924 bei Taungs im Betschuanaland der Kinderschädel gefunden wurde, dem DART die Bezeichnung Australopithecus africanus gab, da zweifelte niemand daran, daß diese Genus-Bezeichnung richtig sei. Denn trotz aller menschlichen Merkmale im Verhältnis von Gehirn- zu Gesichtsschädel und vor allen Dingen trotz des Zahnbogens und Gebisses war die absolute Größe und auch die Form des Schädels so schimpansenähnlich, daß sicher ein Anthropoide und kein Hominide vorlag. Zwar fiel die Größe der ersten Dauermolaren auf, sodaß manche 
Autoren an alte Gorillaverwandtschaft dachten, aber wichtige Merkmale wiesen doch auf die Schimpansenbeziehung hin. DART besprach zwar gleich anfangs die Frage, wie der Schädel wohl im erwachsenen Zustand aussehen möge: ob er mehr zur anthropoiden oder zur hominiden Seite auswachsen würde. Beides konnte möglich sein; aber man war doch mehr der Meinung, daß die hominiden Charaktere sich verstärken würden.

Und die weiteren Entdeckungen haben dieser Meinung recht gegeben. Bekanntlich machte BROOM seit 1936 Entdeckungen, die nicht nur heute, sondern überhaupt in der ganzen Geschichte der Palaeanthropologie wohl das wichtigste sind, was uns je in die Hände gekommen ist. 1856 erregte der Neandertaler-Fund bei Düsseldorf Aufsehen, und 1891 beendete DUBOIS' Endeckung des Pithecanthropus die alte Frage nach dem missing-link. Aber beide Funde ließen sich einordnen, und spätere Entdeckungen beseitigten alle noch verbliebenen Zweifel. Aber was seit 1936 in Südafrika ausgegraben oder vielmehr aus dem Kalkstein herausgesprengt wurde, machte das Problem noch verwirter - falls man nicht in dieser Tatsache selbst seine Lösung erblicken will. Denn man weiß jetzt wirklich nicht, ob man die Schädel als anthropoid oder hominid ansehen und damit auf die tierische oder menschliche Seite stellen soll. Das war natürlich ein Resultat, das zur Bestätigung einer Entwicklungsreihe gehört; und es ist das Wichtige, daß dieses Problem nun wirklich unlösbar vor uns steht.

Obwohl BROOM alle späteren Funde doch zur Australopithecinen-Gruppe stellt - wohin sie zweifellos auch gehören -, so gab er doch den neuen Fundstücken neue Namen. Sie sind als Plesianthropus und Paranthropus bekannt geworden. Hier interessiert wohl vor allen Dingen die Frage der geologischen Einordnung, und sie ist auch mitbestimmend für die Namengebung, denn der Taungsfund wird jetzt als das zeitlich älteste Stück angesehen und an die Grenze vom mittleren zum oberen Pliozän gestellt. Die Plesianthropusgruppe soll mehr in das obere Pliozän gehören und umschließt die Fundstätte von Sterkfontein in Transvaal. Geologisch am jüngsten sind die Paranthropusstücke von Kromdraai und Swartkrans in Transvaal, die in das frühe Pleistozän eingereiht werden.

Nun kann natürlich ein Anthropus (im Sinne von Pithecanthropus = Affenmensch), also immerhin ein Mensch, nicht in eine Pithecus-Gruppe gestellt werden, wobei "Pithecus" hier im Sinne des wissenschaftlichen Namens des Schimpansen "Anthropopithecus" gebraucht wird. Also nicht schlechthin "Affe", sondern „Menschenaffe“ bedeutet. Aber es ist doch möglich! Denn der Ausstralopithecus am Ende des mittleren Miozäns kann sehr wohl ein Pithecus und sein Nachkomme an der Grenze zum Pleistozän oder im frühen Diluvium selbst kann ein Anthropus geworden sein. Es ist ja immer so, daß wir bei einer geologischen Erörterung uns an der Länge der Zeiträume nicht stoßen. Bei den Fossilien selbst haben wir leicht die Neigung, die Zeiten zusammenzuziehen. Bleiben wir aber bei der geologischen Datierung, dann trennen den Paranthropus doch einige oder mindestens eine Million Jahre vom Australopithecus. Also eine Zeit, von der wir uns wirklich keine Begriffe machen können. Es ist dann sehr wohl möglich, daß der Plesianthropus wirklich dicht beim Menschen stand, wie sein Name sagt, daß der Paranthropus im Diluvium bereits a m Menschen v o r bei gegangen war. Nun soll man natürlich bei all den Fundbezeichnungen in der menschlichen Vorgeschichte nicht die strengen Maße der Nomenklatur oder der wörtlichen Übersetzung anlegen; die Hauptsache ist hier, ob die doppelte Genusbezeichnung innerhalb einer Gruppe wirklich gerechtfertigt ist. 
Die geologischen Datierungen sind faunistisch begründet. Wie weit sie berechtigt sind, muß Geologie und Palaeontologie entscheiden. Gerade aus Afrika wird ja mehrfach das lange Überleben altertümlicher Formen gemeldet und ist bis auf den heutigen Tag wirklich bestätigt. Zunächst seien einmal die Funde aus Südafrika genannt, soweit sie uns bisher bekannt geworden sind. Ich habe Herrn Dr. BROOM und Herrn Dr. DART für mehrfache private Benachrichtigungen zu danken.

Es wurden gefunden:

1924 bei Taungs (Betschuanaland) Australopithecus africanus (DART) Schädel 4-5jähriges Kind (type). Mittel - Oberpliozän;

$1947 \mathrm{ff}$ im Makapansgattal Australopithecus prometheus (DART);

1. (a) ein Occiput

(b) ein Cranio-facial-Fragment

- beide wahrscheinlich von demselben weiblichen Schädel -

2. (a) ein Mandibulare

(b) ein linkes Ilium

(c) ein rechtes Ischium-Fragment

(d) Calvarium-Fragment

- alle wahrscheinlich von einem jugendlichen männlichen Fundstück -

3. (a) einzelne Parietal-Knochen

(b) natürlicher Gehirnausguß dieser Knochen

- wahrscheinlich von einem jugendlichen Wesen -

4. ein Maxillare eines alten weiblichen Wesens vorgeschrittenen Alters.

5. ein Maxillare eines Kindes

6. drei einzelne Zähne.

1936-38 bei Sterkfontein (nördlich von Krugersdorp) Plesianthropus transvaalensis (BROOM).

1. S 1 ein fast vollständiger Schädel eines jungen Mannes ohne Mandibulare.

2. S 2 ein gut erhaltenes Maxillare - wahrscheinlich weiblich - .

3. S 3 ein zerbrochener unvollständiger Schädel - wahrscheinlich der eines alten Mannes -

4. S 4 ein beachtlicher 'Teil eines Unterkiefers.

Gefunden nach 1947:

5. S 5 ein vollständiger Schädel - wahrscheinlich der einer erwachsenen Frau.

6. S 6 ein sehr unvollständiger Schädel - wahrscheinlich weiblich - .

7. S 7 ein sehr verdrückter aber leidlich vollständiger Schädel.

8. S 8 ein großer Teil der Schädelbasis mit Teilen des Frontale der Parietalia und des Occipitale.

9. ein Mandibulare, obwohl verdrückt doch nahezu vollständig, mit dem größten Teil der Zähne - wahrscheinlich männlich -

10. Teile der Scapula und das obere Ende eines rechten Humerus.

11. Teile des Beckens und ein Teil des Femur.

1938 bei Kromdraai in Transvaal Paranthropus robustus (BROOM). Alter: wahrscheinlich Pleistozän.

Schädel eines wahrscheinlich männlichen Wesens (zertrümmert). 1948 bei Swartkrans (eine Meile von Sterkfontein entfernt) Paranthropus crassidens (BROOM). Alter: wahrscheinlich oberes Pliozän. 
1. Mandibulare und Zähne.

2. Schädelfragment mit deutlicher Crista sagittalis und einem Gehirnvolumen von $900 \mathrm{ccm}$.

1949 bei Swartkrans (eine Meile von Sterkfontein entfernt) Telanthropus capensis. Alter: wahrscheinlich unteres Pleistozän.

ein Mandibulare (gehört wohl nicht zur Gruppe der Australopithecinen), wird unten noch behandelt.

Die Untersuchungen gehen weiter und werden noch mehr Material liefern. Es ist jetzt überhaupt in Afrika eine glückliche Periode der Entdeckungen auf anthropologisch-paläontologischem Gebiet angebrochen. Das liegt aber nicht etwa am Lande, sondern an der Intensität der Gelehrtenarbeit. Denn das gleiche hatte uns HAUSER in der Dordogne, BLACK und WEIDENREICH bei ChouKou-Tien und v. KÖNIGSWALD auf Java schon einmal vorgemacht. Und wir könnten dasselbe auch bei uns in Deutschland haben, wenn wir die Mittel dafür aufbringen würden; denn die paläontologischen Beweise für die Menschwerdung sind noch wichtig genug.

Um Verwechslungen vorzubeugen, seien daher noch andere afrikanische Funde genannt, die in der Tagespresse häufig mit den genannten Entdeckungen durcheinander gebracht werden. Britisch- und Deutsch-Ost-Afrika sind die Fundstätten von miozänen Vor-Schimpansen, die HOPWOOD als P r oc on s u l bezeichnet hatte (nach einem Varieté-Schimpansen, der "Consul“ hieß). Die ersten Fundstücke bezogen sich auf Unter- und Oberkiefer, die so ähnlich sind, daß sie beinahe aufeinander passen, obwohl sie verschiedenen Individuen angehören. Im Oktober 1948 fand Miss LEAKEY einen wieder hierhin gehörigen Schädel mit ganzem Gesicht und vorderem Schädelteil. Dieser Fund erregte besonderes Aufsehen, weil die Supraorbitalwülste an ihm nicht vorhanden sind, obwohl die Stirn sehr fliehend ist. Als Zeit wird das Miozän angegeben, ohne bisherige noch genauere Datierungen. Es wäre also möglich, daß wir hier Vorfahren des heutigen Schimpansen hätten, die noch nicht so spezialisiert sind. Daß diese Proconsuliden bereits neben Anthropoiden einen Weg zur späteren Hominidenbildung zeigen, ist möglich; aber wir sind nicht in der Lage, im Miozän bereits eine Trennung beider Zweige $\mathrm{zu}$ behaupten.

Zum Rhodesia-Menschen vom Brocken Hill und zum Afrikanthropus vom Njarasasee sind auch vielleicht aufklärende Neuentdeckungen hinzugekommen. Ein Unterkiefer, der am 29. 4. 1949, auch bei Swartkrans, gefunden wurde, erhielt den Namen Telanthropus capensis. Sicher steht er aber dem Menschen garnicht so fern; die Entdecker ROBINSON und BROOM glauben selbst, daß dieses Stück nicht zur Paranthropus-Gruppe gehört, sondern ein Pithecantropus ist. Dazu paßt, daß ich bei meiner Africanthropus-Rekonstruktion einen mutmaßlichen Unterkiefer - von dem ja nichts gefunden wurde - einzeichnete (1936), der völlig dem jetzt gefundenen Telanthropus-Kiefer entspricht. Vielleicht hätte das Stück also den alten Namen "Africanthropus“ erhalten können. Die Zeit soll bei beiden jünger sein, als die zugehörige Stufe erfordert. Das ist natürlich möglich in dem genannten Sinne, daß Afrika geeignet ist, Relikte längere Zeit lebendig zu erhalten. Aber etwas Neues ist jetzt von dort gemeldet worden: Kieferreste und Zähne, die so groß sind, daß sie zum Meganthropus von Java gestellt werden müssen. Der Fund ist ein Stück von der rechten Oberkieferhälfte und enthält beide Prämolaren; es wurde von KOHL-LARSEN auf seiner 2. Expedition 1939 in Ostafrika gefunden und von uns selbst bearbeitet. Da es zur Meganthropus-Gruppe von Java paßt, benannte ich es „Meganthropus 
africanus“ (Ztschr. f. Morphol. u. Anthropol. 1950. Heft 1). Alles das gehört also nicht mit zu, den Australopithecinen; zeigt aber, wie reichhaltig Afrika an Summoprimaten (Gorilla-Schimpanse-Mensch) einmal gewesen ist.

Die Bedeutung der Australopithecinen oder des Plesianthropus und Paranthropus für die menschliche Stammesgeschichte liegt in der Tatsache, daß wir sie mit dem besten Willen nicht mehr widerspruchslos einordnen können. Auch die erwachsenen Stücke, welche DART's Mutmaßungen bestätigten, zeigen in Seitenansicht ganz klare Anthropoiden von Schimpansenform; denn eine solche Schnauzenbildung kann man nicht gut als hominid bezeichnen. Umso verblüffender ist es, daß bei diesem Prognathismus sich ein Zahnbogen und ein Gebiß befindet, das einfach nur als hominid eingeordnet werden muß. Die Zahnreihen sind lückenlos geschlossen, haben also kein Diastema vor oder hinter den Eckzähnen; die Eckzähne selbst sind zwar kräftig, überragen aber nicht das Niveau der anderen Zähne. Die Form der Krone ist schon mehr menschlich (hoch und tulpenförmig) und nicht schimpansisch, niedrig und oben zusammengezogen. Auch weitere Einzelheiten des Zahnreliefs zeigen eine große Mischung von anthropoiden und hominiden Charakteren.

Am Gehirnschädel haben wir kein äffisches Pla n u m nuchale, sondern es ist mehr gerundet, was allerdings auch bei weiblichen Schimpansen der Fall ist. Aber das Hinterhauptsloch ist soweit nach vorn verlagert, daß man mit Sicherheit auf aufrechten Gang schließen kann. Und das wird bestätigt durch die Skelettfunde.

Ich selbst habe schon immer gesagt, daß wir nach den Schädelfunden, die wir aus der Stammesreihe der Menschheit kennen, lieber einmal ein Becken sehen möchten. Denn auch der schönste Schimpanse hat ein so äffisches Becken, daß gar keine Verbindung zum Menschen besteht. Das ist natürlich durch die baumbewohnende Lebensweise verständlich. Nun haben wir von Sterkfontein ein sehr schön erhaltenes Becken, das zum Plesianthropus gestellt wird. Es zeigt k e i n e Übergangsformen, sondern entspricht dem, was wir aus der ganzen Skeletthaltung kannten. Es ist hominid und ähnelt fast ganz der Form des heutigen Buschmanns. Auch die Reste der oberen Extremitäten deuten auf aufrechten Gang hin, obwohl hier zwischen Schimpanse und Mensch nicht so große Unterschiede vorhanden sind.

$\mathrm{Zu}$ allem kommt nun die Lebensweise dieser Australopithecinen hinzu. Sie lebten nicht nur in offenem Gelände und in Höhlen des Kalkgebirges, sondern sie waren auch Jäger und Sammler. Die vielen mitgefundenen und zertrümmerten Pavianschädel müssen als Beutestücke angesehen werden. Und wie bereits gesagt, war auch durch chemische Untersuchung Verkohlung dieser Knochen erkannt worden. Wir müssen also annehmen, daß der Gebrauch naturgegebenen Feuers nicht mehr unbekannt war. Es sind also viele Argumente vorhanden, die einen Namen auf Anthropus rechtfertigen, obwohl die Schädel selbst noch einen menschenäffischen Eindruck machen. Außer BROOM und DART haben verschiedene Autoren zu diesem Problem Stellung genommen; u. a. Le GROS CLARK in Oxford, der die Stücke selbst gesehen hat, HEBERER und SERA. Erinnert sei auch an LINNE, der ja für den Schimpansen den Namen Homo troglodytes gab. Ich selbst schlug als Bezeichnung der Stufe vor: Propithecanthropus. Dann kann man darüber diskutieren, ob ein P r o-Pithecanthropus schon ein Pithecantropus oder noch ein Anthropopithecus ist. Und daß diese Diskussion nicht $\mathrm{zu}$ einem allerseits befriedigendem Ergebnis kommt, liegt an den Fundstücken selbst.

Das ist das Wichtige für unsere Stammesgeschichte. 